\title{
BMJ Open Quality Improving medication optimisation in left ventricular systolic dysfunction after acute myocardial infarction
}

\author{
Paul Forsyth, ${ }^{\oplus 1}$ Lynsey Moir, ${ }^{1}$ lain Speirits, ${ }^{1}$ Steve McGlynn, ${ }^{1}$ Margaret Ryan, ${ }^{1}$ \\ Anne Watson, ${ }^{2}$ Fiona Reid, ${ }^{2}$ Christopher Rush, ${ }^{3}$ Clare Murphy ${ }^{3}$
}

To cite: Forsyth $\mathrm{P}$, Moir L, Speirits I, et al. Improving medication optimisation in left ventricular systolic dysfunction after acute myocardial infarction. BMJ Open Quality 2019;8:e000676. doi:10.1136/ bmjoq-2019-000676

Received 11 March 2019 Revised 23 July 2019 Accepted 24 July 2019
Check for updates

(C) Author(s) (or their employer(s)) 2019. Re-use permitted under CC BY-NC. No commercial re-use. See rights and permissions. Published by BMJ.

${ }^{1}$ Pharmacy Services, NHS Greater Glasgow and Clyde, Glasgow, UK

${ }^{2}$ Pharmacy, NHS Education for Scotland, Glasgow, UK

${ }^{3}$ Cardiology, Royal Alexandra Hospital, Paisley, , UK

Correspondence to Paul Forsyth, Pharmacy Services, NHS Greater Glasgow \& Clyde, Glasgow G76 7AT, UK; paul.forsyth@nhs.net

\section{ABSTRACT}

Glasgow city has the highest cardiovascular disease (CVD) mortality rate in the UK. Patients with left ventricular systolic dysfunction after acute myocardial infarction represent a 'high-risk' cohort for adverse CVD outcomes. The optimisation of secondary prevention medication in this group is often suboptimal. Our aim was to improve the use and target dosing of ACE inhibitors (ACEI), angiotensin II receptor blockers (ARBs) and beta-blockers in such patients, through pharmacist-led clinics and cardiology multidisciplinary team collaboration. Retrospective audits characterised baseline care. Prospective pharmacist-led clinics were piloted and rolled out across seven hospitals and primary care localities over four Plan-Do-Study-Act cycles. 'Hub' and 'spoke' clinics utilised independent prescribing pharmacists with different levels of cardiology experience. Pharmacists were trained through a bespoke training programme- 'Teach and Treat'. Consultant cardiologists provided senior support and governance. Patients attending prospective pharmacist-led clinics were more likely to be prescribed an ACEI (or ARB) and beta-blocker $(n=856 / 885(97 \%)$ vs $n=233 / 255(91 \%)$, $p<0.001$ and $n=813 / 885$ (92\%) vs $n=224 / 255(88 \%)$, $p=0.048$, respectively) and be on target dose of ACEI (or ARB) and beta-blocker ( $n=585 / 885(66 \%)$ vs $n=64 / 255$ (25\%), $p<0.001$ and $n=218 / 885$ (25\%) vs $n=17 / 255$ $(7 \%), p<0.001$, respectively) compared with baseline. The mean dose of ACEl (or ARB) and beta-blocker was also higher $(79 \%$ vs $48 \%$ of target dose, $p<0.001 \%$ and $48 \%$ vs $33 \%$ of target dose, $p<0.001$, respectively) compared with baseline. Use of secondary prevention medication was significantly improved by pharmacist and cardiology collaboration. These improvements were sustained across a 4-year period, supported by a novel approach called 'Teach and Treat' which linked training to defined clinical service delivery. Further work is needed to assess the impact of the programme on long-term CVD outcomes.

\section{PROBLEM}

National Health Service (NHS) Greater Glasgow and Clyde is Scotland's largest regional health authority, delivering primary, secondary and tertiary care to approximately 1.2 million residents. It encompasses seven local acute and ambulatory hospitals and six separate local council authorities. Three out of these six council authorities appear in the top 10 list of UK-wide authorities with the highest age-standardised death rates for cardiovascular disease (CVD), with Glasgow City having the highest overall rate with approximately 400 age-standardised deaths per 100000 population. ${ }^{1}$ Scotland also has amongst the highest prevalence of coronary heart disease across the UK. ${ }^{1}$ Despite declining case fatality rates from myocardial infarction (MI) across the $\mathrm{UK},{ }^{23}$ patients have worse outcomes than those from comparable European countries. ${ }^{4}$

The optimisation of secondary prevention medication is crucial in improving outcomes for post-MI patients, especially in those with left ventricular systolic dysfunction (LVSD) ${ }^{5-7}$ ACE inhibitors (ACEI), or angiotensin II receptor blockers (ARB) if the patient is intolerant, and beta-blockers are first-line agents for such patients and should be prescribed in all patients unless contraindicated. ${ }^{7}$ These medications should be optimised early in the postdischarge phase, ideally utilising non-medical prescribers. ${ }^{78}$

Within NHS Greater Glasgow and Clyde, baseline acute MI care involved patients being initiated on ACEI (or ARB) and betablocker during their admission. These medications were then reviewed postdischarge by cardiac rehabilitation nurses and recommendations were made to general practitioners (GPs) about medication optimisation goals as part of a broader review. Cardiologist outpatient clinic follow-up typically took place 6-12 months postdischarge. Patients with post-MI LVSD without overt clinical heart failure (HF) were not routinely reviewed by HF nurses.

At cardiologist-led clinic follow-up visits, it was noted that many patients were not being prescribed evidence-based therapy at fully optimised doses. Therefore, a baseline audit of care data was conducted in two local hospitals to characterise care-Royal Alexandra Hospital and Vale of Leven. Data were collected through retrospective audit of the cardiac rehabilitation databases and 
electronic patient records for all consecutive patients with incident acute MI and moderate or severe LVSD on echocardiography over 12 months (1 September 2012-31 August 2013). The endpoints were: the percentage of patients treated with, mean percentage of European Society of Cardiology target dose $^{5}$ achieved and percentage of patients on full target dose of ACEI/ARB and beta-blocker at the end of the formal cardiac rehabilitation programme. The target daily doses used were: ramipril $10 \mathrm{mg}$, enalapril $20 \mathrm{mg}$, lisinopril $20 \mathrm{mg}$, candesartan $32 \mathrm{mg}$, losartan $150 \mathrm{mg}$, valsartan $320 \mathrm{mg}$, bisoprolol $10 \mathrm{mg}$, carvedilol $50 \mathrm{mg}$ and nebivolol $10 \mathrm{mg}$.

Fifty-seven patients with moderate or severe post-MI LVSD were identified. Baseline overall use of ACEI (or ARB) was $89 \%(n=51 / 57)$. The mean percentage of ACEI (or ARB) target dose was $44 \%$, with $21 \%(\mathrm{n}=12 / 57)$ of patients achieving the full target dose. Baseline use of beta-blocker was $82 \%(n=47 / 57)$. The mean percentage of beta-blocker target dose was $31 \%$, with $7 \%(n=4 / 57)$ of patients achieving the full target dose.

The Specific Measurable Achievable Realistic Time-limited aim of our programme was to improve the use and target dosing of ACEI (or ARB) and beta-blocker in all patients with LVSD secondary to incident acute MI across a large regional health authority by the end of a patient's cardiac rehabilitation programme, typically within 4 months of discharge from hospital, through pharmacist-led clinics and cardiology multidisciplinary team collaboration.

\section{BACKGROUND}

The development of LVSD independently predicts mortality in patients after acute MI and, therefore, identifies a 'high-risk' cohort. ${ }^{9}$ LVSD increases the risk of both future $\mathrm{HF}$ and sudden cardiac death. ${ }^{9}$

Disease registry data shows us that the optimisation of secondary prevention medication is known to be suboptimal across many healthcare systems. ${ }^{9}$ Improving HF and MI outcomes are key areas for service improvement across the UK. ${ }^{1011}$ Heart disease management and rehabilitation are also key priorities for Scottish Government. ${ }^{12}$

Since 2006, pharmacists and nurses in the UK have been able to train as independent prescribers. A recent meta-analysis shows that such professionals can use these qualifications to deliver aspects of CVD management, such as systolic blood pressure and low-density lipoprotein cholesterol control, comparable with their medical colleagues. ${ }^{13}$ Trial evidence also shows that outcomes can be improved by incorporation of these professionals into the cardiology multidisciplinary team, especially in the field of HF and/or LVSD. ${ }^{1415}$

\section{MEASUREMENT}

\section{Endpoints}

Percentage of patients treated with, mean percentage of target dose $\mathrm{e}^{16}$ achieved of, and percentage of patients on full target dose of ACEI (or ARB) and beta-blocker at the end of the cardiac rehabilitation programme.

\section{Data collection and analysis}

Prospective data on medication optimisation from pharmacist-led clinics were collected on standardised clinic discharge forms and collated in a password-protected Microsoft Access database on NHS-encrypted computers.

Data on the prescribing of medication were summarised in percentages. Data on target doses of medication were summarised in percentage of patients on graded categorical thresholds (ie, $0 \%, 1 \%-24 \%, 25 \%-49 \%, 50 \%-74 \%$, $75 \%-99 \%$ and $100 \%$ of target dose) and mean percentage of target dose achieved across all patients. The $\chi^{2}$ tests and t-tests were used to test for differences in the endpoints at the end of the programme compared with baseline.

\section{DESIGN}

In collaboration with consultant cardiologists, pharmacist-led post-MI LVSD clinics were designed to improve medication optimisation. Prospective patients were identified during acute MI admissions by cardiac rehabilitation nurses and electronically referred at hospital discharge to the pharmacist-led clinic in addition to all aspects of usual care, including cardiac rehabilitation and cardiologist follow-up. The pharmacists delivering the clinics prescribed in line with guidelines. ${ }^{5616}$ Consultant cardiologists provided senior medical support and associated clinical governance. Patients were vetted to confirm suitability before being invited to attend. Patients were phoned to attend by a service administrator and sent a letter confirming the appointment. Patients not contactable by phone were appointed by letter only. Appointment slots were $15 \mathrm{~min}$ and medical records staff supported clinic administration tasks. Hand-written prescriptions were issued directly from clinics and patients were typically reviewed at 2 weekly intervals until medications were judged to be optimised. Letters to GPs were written within three working days of each clinic, documenting all changes in prescribing. Letters were automatically uploaded to electronic medical records, which were available to all primary and secondary care clinicians.

\section{Ethics}

Ethical approval was not required for this service development project.

\section{Patient and public involvement}

No patients were involved at the design stage. A qualitative patient experiences study has been completed and awaits publication.

\section{STRATEGY}

The service was sequentially rolled out and evaluated over four Plan-Do-Study-Act (PDSA) audit cycles. 
PDSA Cycle 1 (1 September 2013-31 August 2014)

PLAN: Clinics were piloted in two hospitals: Royal Alexandra Hospital and Vale of Leven. This pilot concentrated on patients with moderate or severe LVSD and was supported by funding from NHS Education for Scotland.

DO: Two pharmacists (NHS Agenda for Change (AFC) Band 8a) with between 5 and 9 years of cardiology experience, who were already qualified independent prescribers and trained in clinical examination and venepuncture, delivered the clinics in an outpatient setting.

\section{PDSA Cycle 2 (1 September 2014-31 August 2015)}

$P L A N$ : Clinics were rolled out to two further hospitals: West Glasgow Ambulatory Care Hospital and Glasgow Royal Infirmary. Retrospective baseline data were collected from Glasgow Royal Infirmary for the 6 months preceding initiation of the clinic to ensure that there was a similar requirement for the clinic. No baseline data were collected in West Glasgow Ambulatory Care Hospital, as the original site of this hospital closed during this period making consecutive patient data collection impossible. To support the roll-out and criteria widening (to all grades of LVSD), the model was developed into 'hub' and 'spoke' clinics. Pharmacist independent prescribers with different levels of cardiology experience were recruited to deliver stratified clinics depending on the risk profile of the patient. The risk profile was assessed by the experienced PDSA Cycle 1 pharmacists at the point of vetting. In each hospital site, specialist cardiology pharmacists were used to deliver a 'hub' clinic for patients with moderate or severe grades of LVSD, or those with additional risk factors (eg, signs and symptoms of ischaemia, significant residual coronary heart disease or complex comorbidities). In surrounding primary care localities, such as local health centres, General Practice (GP)-based pharmacists were used to deliver 'spoke' clinics for patients with mild LVSD. GP-based pharmacists were general clinicians and were peer supported remotely by specialist cardiology pharmacists. This phase of the project was supported by funding from NHS Education for Scotland.

DO: Two additional specialist pharmacists were used, both already experienced prescribers with 9-25 years of cardiology experience (AFC Bands $8 \mathrm{a}$ and $8 \mathrm{~b}$ ). An additional nine generalist GP-based pharmacists (AFC Band 8a) were trained to deliver primary care clinics through a bespoke local education and training programme'Teach and Treat'. This programme included experiential learning, taught lectures, online webinars, links to national guidelines, physical examination training (including blood pressure/pulse measurement, pitting for oedema and chest auscultation) and venepuncture. At the end of the training, all pharmacists sat a multiple-choice examination and an objective structured clinical examination, developed in partnership between the experienced PDSA Cycle 1 pharmacists and a consultant cardiologist, to assess competence before commencing autonomous clinic work.
PDSA Cycle 3 (1 September 2015-31 August 2016)

$P L A N$ : Clinics were rolled out to two further hospital sites: New Victoria Infirmary and Queen Elizabeth University Hospital. As before, retrospective baseline data were collected from both sites for the preceding 12 months. The Queen Elizabeth University Hospital opened during this time period but baseline data were able to be characterised from its predecessor, the Southern General Hospital. Unlike the other sites, the Southern General Hospital provided a consultant cardiologist-led post-MI clinic approximately 1 month postdischarge at baseline.

DO: An additional specialist pharmacist IP (AFC Band 7 ), with 14 years of cardiology experience, was trained and deployed in one of the 'hub' clinics. The other new specialist 'hub' clinic was delivered by an existing service pharmacist. An additional two generalist GP-based pharmacists (AFC Band 8a) were trained to deliver primary care clinics. All were independent prescribers.

\section{PDSA Cycle 4 (1 September 2016-31 August 2017)}

PLAN: Clinics were rolled out to the final hospital site: Inverclyde Royal Hospital. Baseline data were not available from Inverclyde Royal Hospital due to a lack of a baseline cardiac rehabilitation database.

DO: Two additional specialist pharmacists (AFC Bands 7 and $8 \mathrm{~b}$ ), with 6 and 19 years of cardiology experience, respectively, and two additional generalist GP-based pharmacist (AFC Band 8a) were recruited to deliver 'hub' and 'spoke' clinics, respectively. All pharmacists were trained as described above and were independent prescribers.

\section{RESULTS}

\section{PDSA Cycle 1}

STUDY: 51 patients were reviewed in the pharmacist-led clinic during the first year of the project. The use of ACEI (or ARB) increased from $89 \%$ in the baseline audit $(\mathrm{n}=51 / 57)$ to $94 \%(\mathrm{n}=48 / 51)$. The mean percentage of ACEI (or ARB) target dose also increased from $44 \%$ in the baseline audit to $72 \%$, with $57 \%(\mathrm{n}=29 / 51)$ patients achieving the full ACEI (or ARB) target dose compared with $21 \%$ in the baseline audit $(n=12 / 57)$. The use of beta-blockers increased from $82 \%$ in the baseline audit $(n=47 / 57)$ to $96 \%(n=49 / 51)$. The mean percentage of beta-blocker target dose also increased from $31 \%$ in the baseline audit to $56 \%$, with $29 \%(\mathrm{n}=15 / 51)$ of patients achieving the full beta-blocker target dose, compared with $7 \%(n=4 / 57)$ in the baseline audit. The results were published in abstract form. ${ }^{17}$

ACT: The service steering group in collaboration with the local strategic cardiology planning committee endorsed a plan to roll the clinics out across all hospital sites in the health authority and to widen the inclusion criteria to include all grades of LVSD post-MI.

\section{PDSA Cycle 2}

STUDY: 58 patients with any grade of LVSD post-MI were identified in the baseline Glasgow Royal Infirmary audit. Ninety-one per cent $(n=53 / 58)$ of Glasgow Royal 
Infirmary patients were prescribed an ACEI (or ARB). The mean percentage of ACEI (or ARB) target dose was $48 \%$, with $24 \%(\mathrm{n}=14 / 58)$ of patients achieving the full target dose. Ninety-one per cent $(n=53 / 58)$ of Glasgow Royal Infirmary patients were also prescribed betablockers. The mean percentage of beta-blocker target dose was $33 \%$, with $7 \%(n=4 / 58)$ of patients achieving the full target dose.

A further 143 patients were reviewed at the pharmacist-led clinics during the second year of the project, making the running total 194 patients. The use of ACEI (or ARB) was 95\% ( $\mathrm{n}=185 / 194)$. The mean percentage of ACEI (or ARB) target dose increased from $72 \%$ in PDSA Cycle 1 to $77 \%$, with $65 \%(n=126 / 194)$ of patients achieving the full ACEI (or ARB) target dose. The use of beta-blockers dipped slightly from $96 \%$ in PDSA Cycle 1 to $93 \%(n=180 / 194)$. The mean percentage of betablocker target dose decreased slightly from $56 \%$ in PDSA Cycle 1 to $52 \%$, with $28 \%$ ( $\mathrm{n}=54 / 194)$ patients achieving the full beta-blocker target dose.

ACT: The service steering group endorsed the plan to continue sequential roll-out across the regional health authority.

\section{PDSA Cycle 3}

STUDY: 76 patients with any grade of LVSD post-MI were identified in the baseline New Victoria Infirmary audit and 64 patients were identified in the Southern General Hospital audit. 88\% ( $\mathrm{n}=67 / 76)$ of New Victoria Infirmary patients and $97 \%(\mathrm{n}=62 / 64)$ of Southern General Hospital were prescribed an ACEI (or ARB). The mean percentage of ACEI (or ARB) target dose was $42 \%$ for New Victoria Infirmary and $61 \%$ for Southern General Hospital, with $18 \%(\mathrm{n}=14 / 76)$ of patients achieving the full target dose at New Victoria Infirmary and 38\% $(\mathrm{n}=24 / 64)$ at Southern General Hospital. Eighty-eight per cent $(n=67 / 76)$ of New Victoria Infirmary patients and $89 \%(n=57 / 64)$ of Southern General Hospital were prescribed beta-blockers. The mean percentage of betablocker target dose was $28 \%$ at New Victoria Infirmary and $41 \%$ at Southern General Hospital, with $3 \%(n=2 / 76)$ of New Victoria Infirmary patients and $11 \%(n=7 / 64)$ of Southern General Hospital patients achieving the full target dose. Baseline medication optimisation in the Southern General Hospital was higher than other sites, potentially due to the baseline consultant cardiologist-led post-MI clinic.

A further 260 patients were reviewed at the pharmacist-led clinics during the third year of the project, making the running total 454 patients. The use of ACEI (or ARB) was $97 \%(n=440 / 454)$. The mean percentage of ACEI (or ARB) target dose increased from $77 \%$ in PDSA Cycle 2 to $80 \%$, with $68 \%(\mathrm{n}=307 / 454)$ patients achieving the full ACEI (or ARB) target dose. The use of beta-blockers was 92\% ( $\mathrm{n}=416 / 454)$. The mean percentage of beta-blocker target dose was $51 \%$, with $29 \% \quad(\mathrm{n}=130 / 454)$ patients achieving the full beta-blocker target dose.
ACT: The service steering group endorsed the plan to continue sequential roll-out to the final hospital site.

\section{PDSA cycle 4}

STUDY: A further 431 patients were reviewed at the pharmacist-led clinics during the fourth year of the project, making the running total 885 patients. Overall use of ACEI (or ARB) was 97\% $(n=856 / 885)$. The mean percentage of ACEI (or ARB) target dose was $79 \%$, with $66 \%(\mathrm{n}=585 / 885)$ patients achieving the full ACEI (or ARB) target dose. The use of beta-blockers was $92 \%$ $(n=813 / 885)$. The mean percentage of beta-blocker target dose dipped slightly from $51 \%$ in PDSA Cycle 3 to $48 \%$, with $25 \%(\mathrm{n}=218 / 885)$ patients achieving the full beta-blocker target.

ACT: The service steering group planned to consolidate the clinic within each hospital site, embedding it in routine care delivery, without any immediate plans for further expansion within the health authority. Discussions were undertaken with Scottish Government about rolling the clinics out to other Scottish health authorities and funding was secured from NHS Education for Scotland to allow sequential national expansion of the 'Teach and Treat'programme.

The table 1 summarises the baseline audits and four PDSA cycle results. The figures 1 and 2 show medication optimisation over the length of the programme compared with the combined baseline audits across the five hospitals.

Patients in the pharmacist-led clinics were statistically more likely to be prescribed ACEI (or ARB) and betablocker compared with patients in the combined baseline audits $(\mathrm{n}=856 / 885$ (97\%) vs $\mathrm{n}=233 / 255$ (91\%), $\mathrm{p}<0.001$ and $\mathrm{n}=813 / 885$ (92\%) vs $\mathrm{n}=224 / 255 \quad(88 \%)$, $\mathrm{p}=0.048$, respectively). Patient's in the pharmacist-led clinics were also more likely to be on full target dose ACEI (or ARB) and beta-blocker compared with baseline $(\mathrm{n}=585 / 885(66 \%)$ vs $\mathrm{n}=64 / 255(25 \%), \mathrm{p}<0.001$ and $\mathrm{n}=218 / 885(25 \%)$ vs $\mathrm{n}=17 / 255(7 \%), \mathrm{p}<0.001$, respectively). The mean dose of ACEI (or ARB) and betablocker was also higher compared with baseline $(79 \%$ vs $48 \%$ of target dose, $\mathrm{p}<0.001 \%$ and $48 \%$ vs $33 \%$ of target dose, $\mathrm{p}<0.001$, respectively).

\section{LESSONS AND LIMITATIONS}

Significant improvements in the important clinical surrogate marker of secondary prevention prescribing were seen over this 4-year programme. Patients with LVSD as a result of an acute MI need to be aggressively managed, as adverse cardiac remodelling can rapidly develop in the post-acute phase of the myocardial insult. ${ }^{18}{ }^{19}$ Evidence shows that early pharmacological optimisation can impact favourably on such adverse remodelling. ${ }^{19}$ Thus, early optimisation of medication in this programme gives patients the best chance of left ventricular recovery and the prevention of HF. 


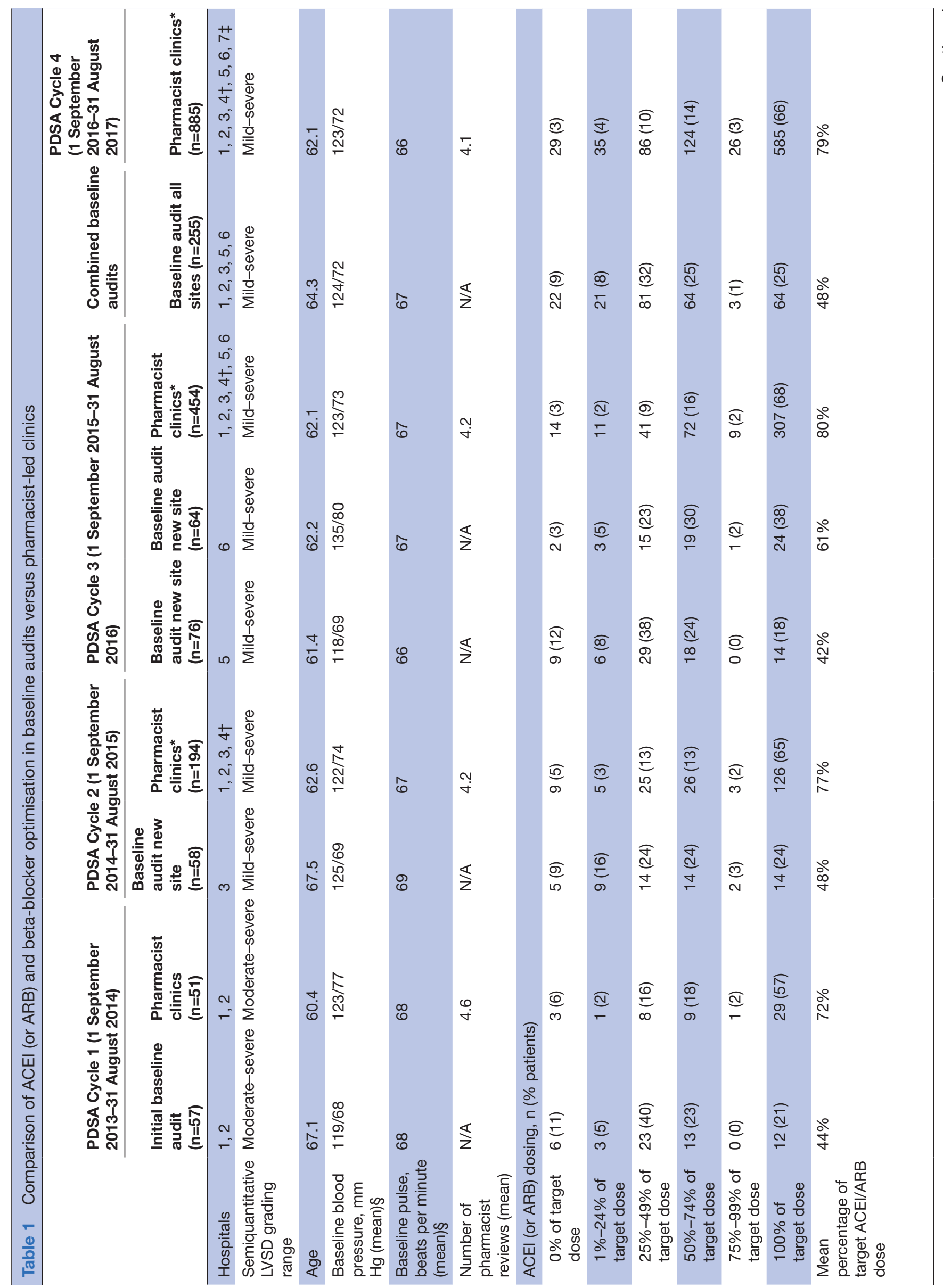




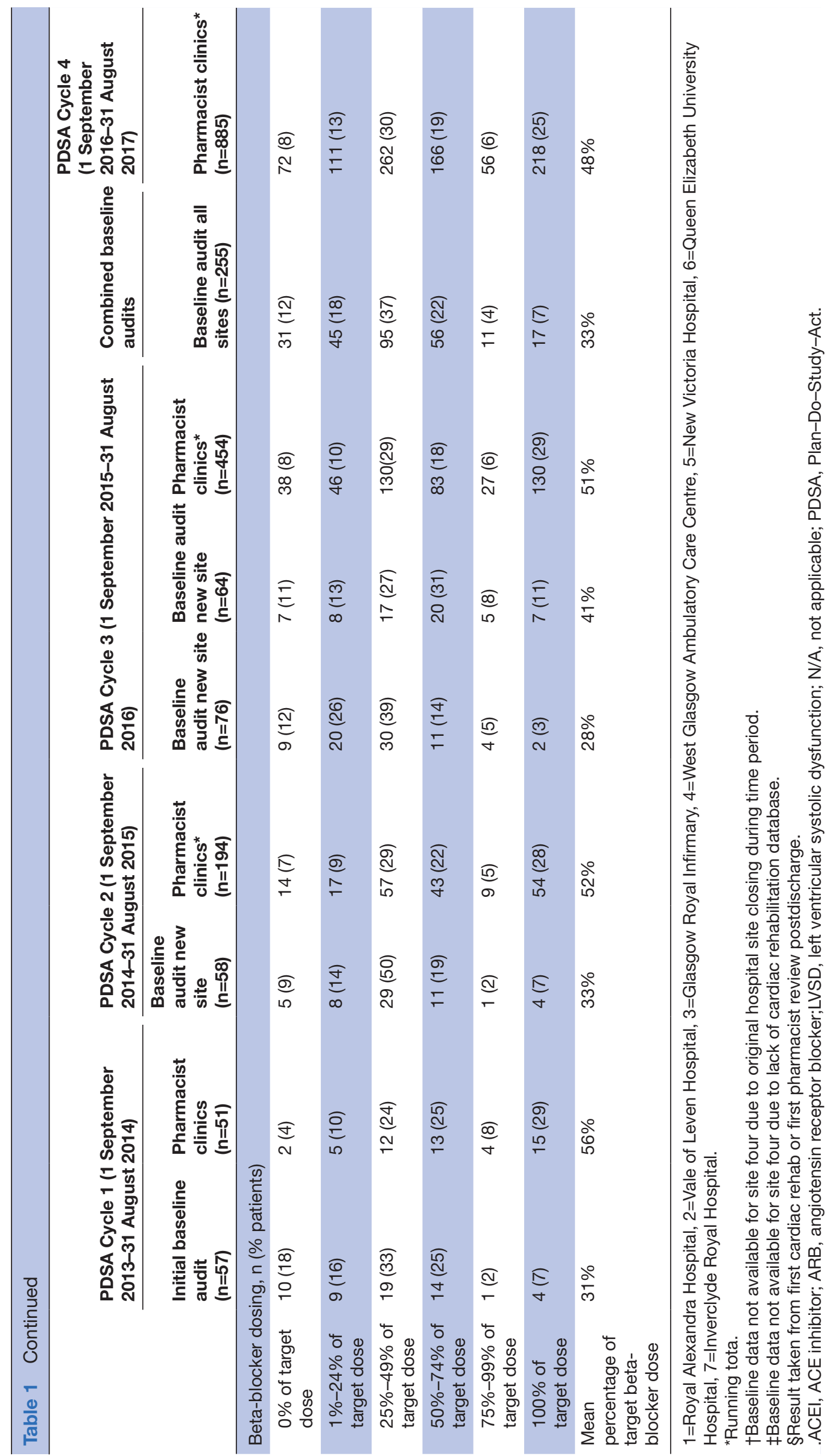




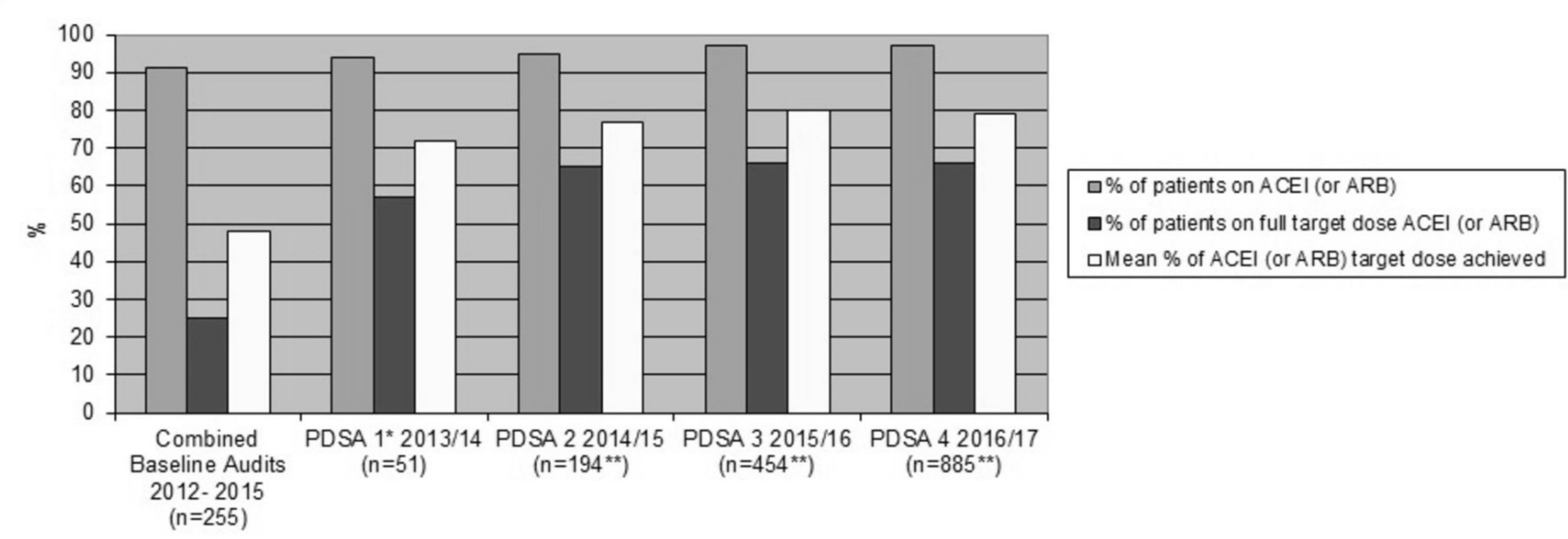

Figure 1

ACEI (or ARB) optimisation over the length of the programme compared to baseline.

*Moderate to severe left ventricular systolic dysfunction patients only; **running total; ACEI, ACE inhibitor; ARB, angiotensin receptor blocker; PDSA, Plan-Do-Study-Act.

Suboptimal uptitration of secondary prevention medication following acute $\mathrm{MI}$ is not a problem unique to NHS Greater Glasgow and Clyde. Evidence from the UK and international healthcare systems highlights the need for further work. ${ }^{20-27}$ The optimisation of ACEI (or ARB) and beta-blocker seen in this programme is superior to that described in previous studies and, therefore, some of the learning may be applicable to other areas. ${ }^{25-27}$

The involvement of pharmacist independent prescribers as care providers in cardiac rehabilitation programmes is a growing role globally. ${ }^{212829}$ It is recognised from within pharmacy that for such interventions to succeed they need to be collaborative and multidisciplinary in nature, rather than standalone systems. ${ }^{15}$ Multidisciplinary models of care, anchored by senior medical leadership, are accepted standards of care,$^{5-8}$ and such working within our programme delivered a clearly defined and robust clinical governance structure, generating widespread trust and confidence in all staff members.

This project was supported by initial NHS funding, designed to support the Scottish Government pharmacy strategy. ${ }^{30}$ Without this funding, senior local strategic pharmacy support and the underpinning government policy, this service would not have been sustainable. Commitment of the pharmacy teams involved was also supported by a redesign in workload, including utilising better skill mix and rationalising the necessity of historic tasks.

\section{Strengths}

This is the first UK programme to systematically describe the post-discharge optimisation of secondary prevention

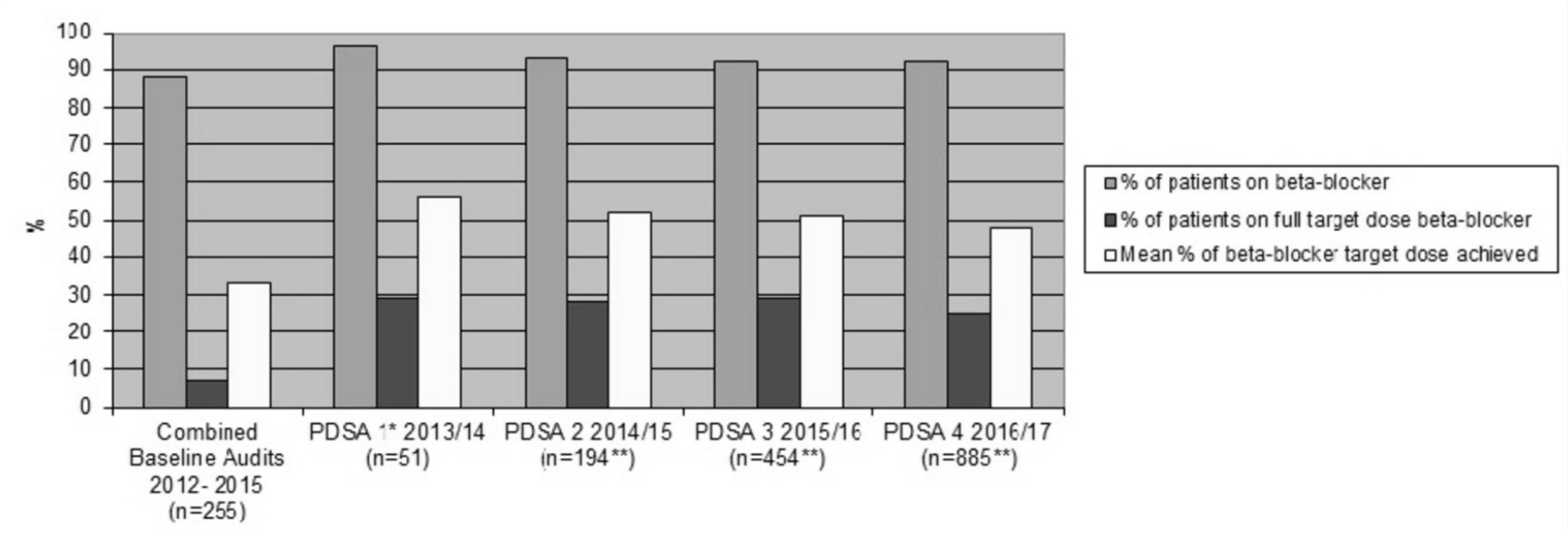

Figure 2

Beta-blocker optimisation over the length of the programme compared to baseline.

*Moderate to severe left ventricular systolic dysfunction patients only; **running total; PDSA, Plan-Do-Study-Act. 
in patients with post-MI LVSD across a large regional health authority. In doing so, it sets a benchmark for comparison for future clinicians and researchers.

The delivery and evaluation of complex interventions is notoriously difficult. ${ }^{31}$ This quality improvement programme delivered lasting change over Scotland's largest health authority, utilising multiple different healthcare professionals across primary and secondary care. The collaborative 'hub' and 'spoke' model used, incorporating both generalists and specialists, has the potential to be replicated by others.

Across Scotland, independent prescribing qualifications are underutilised by pharmacists; this represents an untapped resource. ${ }^{32}$ The 'Teach and Treat' model was implemented as a means of supporting clinical supervision for this group to improve competence and confidence in autonomously managing patients. This type of project, where targeted at measurable public health problems and supported by appropriate governance structures, has the potential to improve clinical outcomes and frontline service delivery. As such, this project is now cited in the Scottish Government strategy for pharmacy as a potential future model of care for the profession. ${ }^{30}$

The work burden for medical staff, including consultants and GPs, is growing. ${ }^{33} 34$ The ageing population and increasing multimorbidity is likely to worsen this problem. This model has the scope to spread prescribing and medication optimisation roles, traditionally delivered by medical staff, across other disciplines.

\section{Limitations}

Some of the limitations of the baseline model of care may not be applicable to other regions. For example, our baseline cardiac rehabilitation model of care did not involve nurse-led prescribing, as seen in some other authorities. Therefore, our findings may not be the best solution in such areas.

The 'hub' clinic model does require patients to attend pharmacist-led clinics at the hospital, in parallel to the conventional cardiac rehabilitation programme, and appointment burden is known to impact significantly on cardiac patients. ${ }^{35}$ A study of qualitative patient feedback has been completed to address these concerns and awaits publication. Evolution of the intervention across PDSA Cycles 2-4 also involved reviewing 'lower risk' patients closer to home, in local health centres, to partially address this issue and in keeping with Scottish Government health policy. ${ }^{36}$

As a complex intervention, the clinic model consists of multiple new components, including the introduction of pharmacists as caregivers, the direct prescribing of medications from clinics (rather than making recommendations to GPs) and an enhanced engagement model utilising phone calls in addition to letters. It is not possible to know the weight of influence of each of the components on the outcome.

As a quality improvement project, rather than a randomised controlled trial, this project is inherently at greater risk of confounding. For example, the patient characteristics in each of the baseline audits and PDSA cycles are likely to differ. This may be reflected in the slight changes in ACEI (or ARB) and beta-blocker prescribing seen over time. It is uncertain whether this is explained by the difference in the patient cohort over time, such as patients with more severe LVSD in the pilot phase having a higher sympathetic drive and hence tolerating slightly higher doses of beta-blocker. Alternatively, changes may reflect the challenges of involving more staff members with different levels of experience. There were also significant changes in our health authority over the time period of the intervention, including the closure of three older hospitals and the opening of a new state-ofthe-art hospital to provide acute care to a large proportion of the population. Reasons for not achieving target doses were not available for both time periods, so are not described.

The pharmacists involved in clinic delivery also commonly impacted on mineralocorticoid receptor antagonist optimisation, as seen in the pilot. ${ }^{17}$ Complete data, including the presence or not of heart failure symptoms and diabetes, needed to compare these endpoints across the whole population were not available in all cardiac rehabilitation databases used for the baseline audits. All patients were also not eligible for these medications (eg, those with mild LVSD). Other clinical factors, including patient education, cardiac rehabilitation engagement, medicines reconciliation, lipid control and the requirement for reassessment of left ventricular function (where appropriate), were also commonly reviewed. Similarly, complete data were not available in the baseline audits in order to describe and compare these interventions, and some of these interventions were only appropriate in subsets of patients.

This paper only describes the prescribing outcomes of patients that were known to the local cardiology teams and engaged with the cardiac rehabilitation process after discharge. It is well known that many patients do not engage fully with cardiac rehab after discharge. ${ }^{37}$ The Inverse Care Law suggests that this group of patients may ultimately have the greatest unmet need. ${ }^{38}$

\section{Next steps}

This paper describes the short-term optimisation of ACEI (or ARB) and beta-blockers in patients with LVSD as a result of an acute MI. Future work is needed to describe the persistence of prescribing over time in these patients. The impact of this project on clinical outcomes is also unknown and requires appropriate evaluation. Plans are underway to deliver a sequential national roll-out of the model to other Scottish regional health authorities.

In parallel to this work, the pharmacy profession in Scotland are now systematically delivering frontline care in primary care. ${ }^{39}$ This paradigm change in the working model of the profession brings with it both opportunities and challenges for this project. Ultimately, this change will result in workforce-wide pharmacists delivering these 
types of interventions as part of routine practice. Plans to adequately train, support, ensure governance and evaluate these processes are vital to ensure success.

To support the delivery of these new advanced pharmacist roles within the cardiology multidisciplinary team, a competency framework for pharmacists has been developed $^{40}$ and work is underway to accredit this with the Royal Pharmaceutical Society, in order to standardise the quality and consistency of this type of advanced practice.

\section{CONCLUSION}

The use of secondary prevention medication with prognostic importance was significantly improved by collaboration between pharmacists and the cardiology multidisciplinary team in patients with post-MI LVSD. These improvements were sustained across multiple sites over a 4-year period, as this service was sequentially rolled-out across a large regional health authority. This achievement was underpinned by a novel multidisciplinary approach called 'Teach and Treat' which linked education and training to defined clinical service delivery, including linking specialist and generalist pharmacy practice. Further work is needed to assess the impact of the programme on long-term cardiovascular outcomes.

Contributors PF, AW, FR and CM contributed to the concept/design. PF contributed to the data analysis and is the lead author. PF, LM, IS, SM and CM delivered staff training/teaching and contributed to the project planning. PF and CM contributed to the funding bid. PF, LM, IS and SM contributed to the intervention. LM, IS, SM, $M R, A W, F R, C R$ and $C M$ contributed to the manuscript revision. MR, AW and FR contributed to the strategic workforce support. AW and FR are the funders and contributed to the intervention planning. CR contributed to the pilot data. CM contributed to the clinical/academic supervision.

Funding NHS Education for Scotland (pilot and training).

Competing interests None declared.

Patient consent for publication Not required.

Provenance and peer review Not commissioned; externally peer reviewed.

Open access This is an open access article distributed in accordance with the Creative Commons Attribution Non Commercial (CC BY-NC 4.0) license, which permits others to distribute, remix, adapt, build upon this work non-commercially, and license their derivative works on different terms, provided the original work is properly cited, appropriate credit is given, any changes made indicated, and the use is non-commercial. See: http://creativecommons.org/licenses/by-nc/4.0/.

\section{REFERENCES}

1. Bhatnagar $\mathrm{P}$, Wickramasinghe $\mathrm{K}$, Williams J, et al. The epidemiology of cardiovascular disease in the UK 2014. Heart 2015;101:1182-9.

2. Information Services Division, NHS National Services Scotland, Edinburgh, UK. Scottish Heart Disease Statistics - Year Ending 31 March 2015. Available: http://www.isdscotland.org/Health-Topics/ Heart-Disease/Publications/data-tables.asp [Accessed 12th Feb 2019].

3. Smolina K, Wright FL, Rayner M, et al. Determinants of the decline in mortality from acute myocardial infarction in England between 2002 and 2010: linked national database study. BMJ 2012;344:d8059.

4. Chung S-C, Gedeborg R, Nicholas O, et al. Acute myocardial infarction: a comparison of short-term survival in national outcome registries in Sweden and the UK. Lancet 2014;383:1305-12.

5. Ponikowski P, Voors AA, Anker SD, et al. 2016 ESC guidelines for the diagnosis and treatment of acute and chronic heart failure. Eur Heart J 2016;37:2129-200.

6. Scottish Intercollegiate Guidelines Network (SIGN). Management of chronic heart failure: a national clinical guideline. Edinburgh: sign;
2016. (sign publication No. 147), 2016. Available: https://www.sign. ac.uk/sign-147-management-of-chronic-heart-failure.html [Accessed 12th Feb 2019].

7. Scottish Intercollegiate Guidelines Network (SIGN). Acute coronary syndrome: a national clinical guideline. Edinburgh: sign; 2016. (sign publication No. 148), 2016. Available: https://www.sign.ac.uk/sign148-acute-coronary-syndrome.html [Accessed 12th Feb 2019].

8. Scottish Intercollegiate Guidelines Network (SIGN). Cardiac rehabilitation: a national clinical guideline. Edinburgh: sign; 2016. (sign publication No. 150), 2017. Available: https://www.sign.ac.uk/ sign-150-cardiac-rehabilitation.html [Accessed 12th Feb 2019].

9. Velazquez E, Francis GS, Armstrong PW. An international perspective on heart failure and left ventricular systolic dysfunction complicating myocardial infarction: the VALIANT registry. Eur Heart $J$ 2004:25:1911-9.

10. National Heart Failure Audit (NHFA). National Institute for cardiovascular outcomes research (NICOR), University College London. Available: https://www.ucl.ac.uk/nicor/audits/heartfailure [Accessed 12th Feb 2019].

11. Myocardial Ischaemia National Audit Project (MINAP). National Institute for cardiovascular outcomes research (NICOR), University College London. Available: https://www.ucl.ac.uk/nicor/audits/minap [Accessed 12th Feb 2019].

12. Heart Disease Improvement Plan. Scottish government. crown Copyright, 2014. Available: https://www.gov.scot/publications/heartdisease-improvement-plan [Accessed 12th Feb 2019].

13. Weeks G, George J, Maclure K, et al. Non-Medical prescribing versus medical prescribing for acute and chronic disease management in primary and secondary care. Cochrane Database Syst Rev 2014.

14. McAlister FA, Stewart S, Ferrua S, et al. Multidisciplinary strategies for the management of heart failure patients at high risk for admission: a systematic review of randomized trials. J. Am. Coll. Cardiol 2004:44:810-9.

15. Koshman SL, Charrois TL, Simpson SH, et al. Pharmacist care of patients with heart failure: a systematic review of randomized trials. Arch. Intern. Med 2008;168:687-94.

16. McMurray JJ, Adamopoulos S, Anker SD, et al. Esc Committee for practice guidelines ESC guidelines for the diagnosis and treatment of acute and chronic heart failure 2012: the task force for the diagnosis and treatment of acute and chronic heart failure 2012 of the European Society of cardiology. developed in collaboration with the heart failure association (HFA) of the ESC. Eur. Heart $J$ 2012;33:1787-847.

17. Rush C, Forsyth $\mathrm{P}$, Moir L, et al. Collaborative care with a pharmacist improves optimisation of secondary prevention medication after discharge in patients with left ventricular systolic dysfunction due to acute myocardial infarction. Eur J Heart Fail 2015;17(Suppl 1).

18. Konstam MA, Kramer DG, Patel AR, et al. Left ventricular remodeling in heart failure: current concepts in clinical significance and assessment. JACC Cardiovasc Imaging 2011;4:98-108.

19. Kramer DG, Trikalinos TA, Kent DM, et al. Quantitative evaluation of drug or device effects on ventricular remodeling as predictors of therapeutic effects on mortality in patients with heart failure and reduced ejection fraction. J Am Coll Cardiol 2010;56:392-406.

20. Stewart KJ, Woothipoom P, Townend JN. Achieving the dose: an audit of discharge medication for the secondary prevention of myocardial infarction. Br J Cardiol 2010;17:142-3.

21. Khatib R, Patel N, Laverty U, et al. Re-Engineering the postmyocardial infarction medicines optimisation pathway: a retrospective analysis of a joint consultant pharmacist and cardiologist clinic model. Open Heart 2018;5.

22. Di Martino M, Alagna M, Cappai G, et al. Adherence to evidencebased drug therapies after myocardial infarction: is geographic variation related to hospital of discharge or primary care providers? A cross-classified multilevel design. BMJ Open 2016;6:e010926-4425.

23. Juilliere $Y$, Cambou JP, Bataille V, et al. Heart failure in acute myocardial infarction: a comparison between patients with or without heart failure criteria from the FAST-MI registry. Rev Esp Cardiol.

24. Gasior M, Gierlotka M, Pyka Łukasz, et al. Temporal trends in secondary prevention in myocardial infarction patients discharged with left ventricular systolic dysfunction in Poland. Eur J Prev Cardiol 2018;25:960-9

25. Pedersen SB, Nielsen JC, Bøtker HE, et al. Beta-blocker therapy early after myocardial infarction: a comparison between medication at hospital discharge and subsequent Pharmacy-Dispensed medication. Drugs Real World Outcomes 2016;3:279-88.

26. Goldberger JJ, Bonow RO, Cuffe M, et al. Beta-blocker use following myocardial infarction: low prevalence of evidence-based dosing. Am Heart J;60:435-42.

27. Grall S, Biere L, Le Nezet M, et al. Relationship between betablocker and angiotensin-converting enzyme inhibitor dose and 
clinical outcome following acute myocardial infarction. Circ $J$ 2015;79:632-40.

28. Dorsch MP, Lose JM, DiDomenico RJ. The effect of cardiovascular credentialed pharmacists on process measures and outcomes in myocardial infarction and heart failure. Pharmacotherapy 2014;34:803-8.

29. Budiman T, Snodgrass K, Komatsu Chang A. Evaluation of pharmacist medication education and post-discharge follow-up in reducing readmissions in patients with ST-segment elevation myocardial infarction (STEMI). Ann Pharmacother 2016;50:118-24.

30. Scottish Government. Crown Copyright. Achieving excellence in pharmaceutical care: a strategy for Scotland, 2017. Available: http:// www.gov.scot/Publications/2017/08/4589 [Accessed 12th Feb 2019].

31. Craig P, Dieppe P, Macintyre S, et al. Developing and evaluating complex interventions: the new medical Research Council guidance. BMJ 2008;337:a1655-983.

32. NHS Education for Scotland (NES). Pharmacist prescribing workforce report for NHS Scotland, 2016. Available: https://www.nes.scot.nhs. uk/pharm-wf-report.html

33. Nicholls M. Cardiologists and the burnout scenario. Eur Heart $J$ 2019;40:5-6.
34. Fisher RFR, Croxson CHD, Ashdown HF, et al. Gp views on strategies to cope with increasing workload: a qualitative interview study. Br J Gen Pract 2017;67:e148-56.

35. Gallacher K, May CR, Montori VM, et al. Understanding patients' experiences of treatment burden in chronic heart failure using normalization process theory. Ann Fam Med 2011;9:235-43.

36. Scottish Government. Crown Copyright. Achieving sustainable quality in Scotland's healthcare: a '20:20' vision, 2011. Available: https://www2.gov.scot/Topics/Health/Policy/2020-Vision [Accessed 12th Feb 2019].

37. British Heart Foundation. National audit of cardiac rehabilitation (NACR) annual statistical report 2017, 2018. Available: https://www. bhf.org.uk/informationsupport/publications/statistics/national-auditof-cardiac-rehabilitation-annual-statistical-report-2017 [Accessed 12th February 2019].

38. Hart JT. The inverse care law. Lancet 1971;1:405-12.

39. The 2018 Scottish General Medical Services Contract. Scottish government. crown Copyright, 2017. Available: https://www2.gov. scot/Topics/Health/Services/Primary-Care/GP-Contract [Accessed 12th Feb 2019].

40. Forsyth P, Warren A, Thomson C, et al. A competency framework for clinical pharmacists and heart failure. Int J Pharm Pract 2018;1(8 Suppl. 1). 